Revue d'histoire de l'Amérique française

REVUE D.HISTOIRE DE L'AMÉRIQUE FRANÇAISE

\title{
Inventaire critique des notaires royaux des gouvernements de Québec, Montréal et Trois-Rivières (1663-1764) (suite et fin)
}

\section{André Vachon}

Volume 11, numéro 3, décembre 1957

URI : https://id.erudit.org/iderudit/301849ar

DOI : https://doi.org/10.7202/301849ar

Aller au sommaire du numéro

Éditeur(s)

Institut d'histoire de l'Amérique française

ISSN

0035-2357 (imprimé)

1492-1383 (numérique)

Découvrir la revue

Citer cet article

Vachon, A. (1957). Inventaire critique des notaires royaux des gouvernements de Québec, Montréal et Trois-Rivières (1663-1764) (suite et fin). Revue d'histoire de l'Amérique française, 11(3), 400-406. https://doi.org/10.7202/301849ar d'utilisation que vous pouvez consulter en ligne. 


\section{INVENTAIRE CRITIQUE DES NOTAIRES ROYAUX DES GOUVERNEMENTS DE QUÉBEC, MONTREAL ET TROIS-RIVIÈRES *}

(1663-1764)

(Suite et fin)

\section{Pierre Raimbault}

Pierre Raimbault naquit à Montréal en octobre 1671. Encore jeune, il s'embarqua pour la France avec son père, qui ne voulait pas mourir au pays. En 1696 cependant, Pierre Raimbault revenait dans sa ville natale avec son épouse. ${ }^{18}$

«Le 9 janvier 1697, Pierre Raimbault recevait une commission de notaire royal à Montréal qu'il fit enregistrer un an après $\gg{ }^{19}$ Cette affirmation de l'Archiviste de la Province n'est accompagnée d'aucune référence. Fidèle à la politique que nous avons exposée dans notre introduction, nous n'acceptons cette affirmation que sous réserves, à cause des nombreuses confusions que nous avons rencontrées sur la qualité de certains notaires.

Le premier acte de Raimbault est daté du 17 novembre 1697. Il pratiqua jusqu'en décembre 1726 .

"Pierre Raimbault qui fut tour à tour, ou concurremment, marchand, notaire, conseiller du roi, procureur du roi, subdélégué de l'intendant, lieutenant civil et criminel et seigneur est une figure importante de l'histoire de la métropole canadienne $\gg .^{20}$

Le greffe de Pierre Raimbault est conservé aux Archives judiciaires de Montréal.

* Voir notre Revue, IX : 423-438, 546-561; X: 93-103, 257-263, 381-390; XI : 93-106, 270-276.

18 Les Cahiers des dix, $1943: 255$.

19 RAPQ, 1921-22: 31.

20 E.-Z. Massicotte, cité dans $R A P Q, 1921-22: 31$. 


\section{MAXIME TAILHANDIER}

Maxime Tailhandier dit La Baume était né à Clermont, Auvergne, en 1665. ${ }^{21}$

Le 19 juin 1699, Tailhandier recevait une commission de notaire seigneurial dans la seigneurie de Boucherville.22

«Le 7 août 1702, il était promu notaire royal par l'intendant Champigny $\gg .{ }^{23}$ Cette affirmation de M. J.-E. Roy réfère aux Registres des Ordonnances des Intendants, XXVI: 84. Or cette référence s'avère erronée. Nous n'avons pu retracer cette commission de Tailhandier.

Il est vrai que dans la commission de Antoine Loiseau, successeur de Tailhandier, ce dernier est qualifié de notaire royal. ${ }^{24}$

Tailhandier pratiqua à la fois comme chirurgien et notaire.

Son greffe est déposé aux Archives judiciaires de Montréal. ${ }^{25}$

\section{Michel LePaILleUR}

En 1701, Michel Lepailleur commençait à pratiquer comme notaire à Québec tout en remplissant les fonctions de juge sénéchal de la seigneurie de Lauzon. ${ }^{26}$

«A l'automne de 1702, Lepailleur obtenait une commission de notaire royal à Montréal $\gg .^{27}$ Encore une fois, ce texte n'est appuyé sur aucune référence.

Dans sa commission de second huissier, Lepailleur est dit «nottaire en la prévosté de cetted. ville ». ${ }^{28}$ Le 13 juillet 1722 cependant, Raudot accorde une commission d'huissier audiencier de la juridiction de Montréal au Sieur Puiperous de La Fosse, en remplacement du sieur Lepailleur que ses fonctions de notaire royal et de concierge des prisons empêchent de remplir cet office. ${ }^{29}$ Qu'en est-il au juste ?

21 Tanguay, op. cit., I : 558.

22 RAPQ, 1921-22: 31 .

23 J.-E. Roy, op. cit., I: 210.

$24 \mathrm{APQ}$, Ord. Int., XVIII: 62.

25 Voir Invent. des greffes des not., VIII : 5-99.

26 RAPQ, 1921-22: 32.

27 Ibid.

28 Jug. et délib., IV : 713.

29 APQ, Ord. Int., VIII: 72. 
Michel Lepailleur fut inhumé à Montréal le 10 avril 1733. ${ }^{3 i \prime}$

Les archives judiciaires de Québec et de Montréal se partagent le greffe de Michel Lepailleur. ${ }^{31}$

\section{Nicolas-Augustin Guillet}

Nicolas-Augustin Guillet de Chaumont était fils d'un notaire royal. «En 1727, il fait une couple d'actes quoiqu'il ne fut pas tabellion, puis le 6 novembre 1728 il commence à exercer la profession de notaire royal $\gg{ }^{32}$ Ce texte ne comporte pas de référence.

Nous n'avons retrouvé aucun texte officiel mentionnant ce notaire.

Guillet de Chaumont cessa de pratiquer en 1752 et fut enseveli à Terrebonne en avril $1768 .^{33}$

Son greffe est aux Archives judiciaires de Montréal.34

\section{III}

Le gouvernement de Montréal a donc compté quarante-six notaires royaux dont le cas est certain, et sept autres notaires dont la qualité de notaires royaux demeure douteuse. Dans l'hypothèse où ces sept notaires auraient réellement reçu une commission de notaire royal, le nombre des notaires royaux du gouvernement de Montréal passerait à cinquante-trois. C'est un chiffre assez élevé, si l'on tient compte que, pendant la plus grande partie du XVII ${ }^{\mathrm{e}}$ siècle, Montréal, au point de vue de la justice, dépendit du Séminaire de St-Sulpice. Il reste cependant qu'à Montréal aussi bien que dans les autres gouvernements, certains notaires subalternes ont été qualifiés à tort de notaires royaux. Nous ferons maintenant l'inventaire rapide de ces notaires.

30 RAPQ, 1921-22: 32. et 25 .

$31 \mathrm{APQ}$, Coll. de pièces jud. et not., liasse 70: Invent. des greffes, 24 $32 R A P Q, 1921-22: 40$.

33 Ibid.

34 Voir Invent. des greffes des not., XVI : 97-198. 


\section{JEAN DE ST-PÈRE}

Jean de St-Père, nous dit Tanguay, ${ }^{35}$ fut notaire royal. Il naquit en 1618, à Domelle, en Gastinois, et fut enseveli à Montréal le 25 octobre 1657.

L'acte de sépulture de St-Père le dit notaire royal. ${ }^{36}$

St-Père cependant n'a pu exercer comme notaire royal, puisque la ville de Montréal était alors une seigneurie, et possédait sa propre organisation justicière. D'ailleurs qui aurait nommé St-Père notaire royal ?

Jean de St-Père fut le premier notaire de Montréal. Il pratiqua de façon intermittente de 1648 à 1657 . réal. ${ }^{37}$

Son greffe est déposé aux Archives judiciaires de Mont-

\section{LAMBERT CLOSSE}

Lambert Closse est un autre notaire qu'on a qualifié à tort de notaire royal. M. Hubert Larue, pour un, dit que «Clausse» fut notaire royal. ${ }^{38}$

Closse, pas plus que St-Père, ne pouvait être notaire royal, et pour les mêmes raisons.

Closse fut enseveli à Montréal le 6 février $1662 .{ }^{39}$ Il avait pratiqué de 1651 à 1657 .

Le greffe de Closse, qui se compose de trente actes, est conservé aux Archives judiciaires de Montréal.40

\section{BÉNIGNE BASSET} Paris. ${ }^{42}$

Bénigne Basset, sieur des Lauriers, naquit en $1639,{ }^{41}$ à

C'est après l'assassinat de Jean de St-Père, que Bénigne Basset fut nommé notaire. Il ne pouvait certainement pas être notaire royal à cette date, quoi qu'en dise l'Archiviste de la Pro-

35 Tanguay, op. cit., I : 555.

36 Tanguay, A travers les registres, 39.

37 Voir Invent. des greffes des not., I : 29-32.

38 J.-E. Roy, op. cit., I: 63.

39 Tanguay, op. cit., 43.

40 Voir Invent. des greffes des not., I : 152-154.

41 Tanguay, Dictionnaire généalogique, I : 28.

42 PAPQ, 1921-22: 18. 
vince, qui écrit que «Bénigne Basset [...] exerça à Montréal comme greffier, tabellion, notaire royal de 1657 à $1677[\ldots] \gg .^{43}$

A l'occasion d'un voyage à Montréal, M. de Mésy et Mgr de Laval nommèrent Basset notaire de la juridiction de Montréal. Quelques semaines plus tard, le 28 octobre 1663, le Conseil Souverain ratifiait cette nomination. ${ }^{44}$ Notons qu'il s'agit ici d'une simple commission de notaire subalterne.

C'est donc à tort que Basset se qualifiait de notaire royal.

Basset décéda à Montréal le 5 août $1699 .^{45}$

Son greffe est aux Archives judiciaires de Montréal.46

Antolne AdHémar

Voir l'inventaire des notaires royaux du gouvernement des Trois-Rivières, IX: 258 et suivantes.

\section{CONCLUSION}

A la fin de cet inventaire critique des notaires royaux des trois gouvernements de Québec, Montréal et Trois-Rivières, tirons quelques conclusions générales.

Dans les trois gouvernements, on compte en tout quatrevingt-quatorze notaires royaux dont la qualité soit absolument certaine. Dans l'hypothèse où les vingt-trois notaires dont la qualité reste douteuse, faute de commission, auraient réellement exercé comme notaires royaux, cent dix-sept notaires royaux auraient exercé dans les trois gouvernements. En tenant compte du fait que le même notaire a pu pratiquer dans deux gouvernements, le bilan par gouvernement s'établit comme suit:

Québec: 47 notaires royaux

5 cas douteux.

Trois-Rivières: 10 notaires royaux

12 cas douteux.

Montréal: 46 notaires royaux

7 cas douteux.

43 Ibid.

44 Jug. et délib., I: 33s.

45 RAPQ, 1921-22: 18.

46 Voir Invent. des greffes des not., I : 161-322. 
Il serait également intéressant d'établir quelques statistiques sur l'âge auquel on nommait les notaires royaux. Au risque d'être incomplet, donnons quand même quelques chiffres.

L'âge moyen, à leur nomination, des vingt-trois notaires royaux du gouvernement de Québec dont nous connaissions la date de naissance est de 35.3 ans. Les plus jeunes notaires royaux à être nommés dans le gouvernement de Québec sont Pierre Duquet, 20 ans, Jean Gloria, Jean-Claude Panet et Prisque Marois, 24 ans. Nicolas Huot avait 58 ans lorsqu'il reçut sa commission, Hiché en avait 53 et Auber, 50.

Dans le gouvernement des Trois-Rivières, nous ne connaissons la date de naissance que de trois notaires royaux. Joseph Caron fut nommé à 57 ans. L'âge moyen de ces trois notaires royaux à leur nomination est de 42.7 ans.

L'âge moyen des notaires royaux du gouvernement de Montréal se rapproche de celui des notaires royaux de Québec, soit 36.5 ans, pour dix-sept notaires dont nous connaissons la date de naissance. Mezière fut nommé à 23 ans, Jacques Crevier Duvernay à 24 ans; Tétro et Souste reçurent leur commission à 52 ans.

Ainsi voit-on que, d'une part, le principe voulant qu'on ne nommât pas un notaire avant qu'il eût atteint sa majorité n'était pas tellement rigoureux; et que, d'autre part, on accordait des charges de notaire royal à des gens de tout âge.

Quant aux pays d'origine des notaires, disons que sur soixante-trois notaires royaux dont nous connaissions le lieu d'origine, on compte quarante-quatre Français, dix-huit Canadiens et un Suisse, soit une proportion de Canadiens égale à $28.5 \%$.

Notons enfin que des gens de toutes les conditions pouvaient accéder à une charge de notaire royal: fils de famille, marchands, militaires, gens de métier.

Souvent, ces gens, une fois pourvus d'une commission de notaire royal, continuaient d'exercer leur ancienne profession. On aura remarqué, au cours de cet inventaire, le cumule extra- 
ordinaire des charges de certains notaires: cela provient de ce que les honoraires n'étaient pas très élevés. Autant de questions que nous nous proposons d'étudier dans un travail subséquent. ${ }^{1}$

\section{ANDRÉ VACHON}

Québec, 15 déc. 1954 - 11 mai 1955.

1 Depuis la rédaction de cet Inventaire, qui date de plus de deux ans, l'auteur a publié, dans la Revue de l'Université Laval (novembre 1955), un article, Le notaire en Nouvelle-France, qui reprend et développe les éléments de cette conclusion, et tente de les synthétiser en une image plus riche et plus complète du notaire, type social de la Nouvelle-France. 